NASZA DERMATOLOGIA Online OUR DERMATOLOGY Online

Source of Support: Nil

Competing Interests: None

\section{SARCOIDOSIS PRESENTING ERYTHEMA NODOSUM-LIKE LESIONS: REPORT OF TWO CASES}

\author{
Taeko Nakamura-Wakatsuki, Toshiyuki Yamamoto
}

Department of Dermatology, Fukushima Medical University, Hikarigaoka 1, Fukushima 960-1295, Japan

Corresponding author: Dr. Taeko Nakamura-Wakatsuki

\begin{abstract}
Introduction: Sarcoidosis is a systemic non-infectious granulomatous disorder which exhibits various specific and non-specific cutaneous manifestations. Erythema nodosum-like eruption is a rare specific lesion associated with sarcoidosis, which histologically shows noncaseating epithelioid granuloma. It is suggested that this type is frequently associated with ocular sarcoidosis.

Main observations: We describe two Japanese cases presenting with erythema nodosum-like eruptions on the lower legs, which histologically showed sarcoidal granuloma. The tenderness of erythema nodosum-like eruption is milder than that of non-specific erythema nodosum. Lung involvement was observed in both cases, and ocular involvement was seen in one of them.

Conclusions: Because of the high frequency of extra-cutaneous involvement, we should carry out skin biopsies to reveal specific sarcoidal granulomas appropriately and follow up patients with erythema nodosum-like eruption carefully.
\end{abstract}

Key words: sarcoidosis; erythema nodusum-like eruption; sarcoidal granuloma

\section{Introduction}

Sarcoidosis is a systemic granulomatous disorder of unknown etiology. Approximately, $25 \%$ of patients have cutaneous lesions, which exhibit different manifestations depending on different races [1]. Specific manifestations include plaques, papules, maculopapules, subcutaneous nodules, infiltrative scars, and lupus pernio. EN-like eruption usually occurs on the lower limbs, which histologically shows non-caseating epithelioid granuloma located in the dermis to subcutis. On the other hand, erythema nodusum (EN), calcifications, prurigo, erythema multiforme, etc, are non-specific lesions of sarcoidosis, among which EN is occasionally seen. Skin manifestations might be the initial sign to make the diagnosis of sarcoidosis; however, ENlike lesion is not well-known. We report herein rare cases presenting with EN-like eruptions on the lower legs.

\section{Case Report}

Case 1:

A 30-year-old man complained of 2-weeks' history of erythema on his lower legs. He had been suffering from diarrhea and high fever. In addition, he had been congested with his eyes 1-month before visiting our department. A physical examination showed numerous erythematous patches with mild tenderness on the anterior aspects of bilateral lower legs (Fig. 1a). The histopathological findings revealed non-caseating granulomas with epithelioid cells throughout the dermis (Fig. 1b). The granulomas were associated with minimal infiltration of lymphocytes and plasma cells. Laboratory examination showed increased serum levels of angiotensin-converting enzyme (ACE) (33.7 IU/L, normal 7 to 25 ). Chest X-ray and computed tomography (CT) scan revealed bilateral hilar lymphadenopathy. Gallium scintigraphy confirmed the bilateral hilar involvement. Ophthalmological examination revealed granulated uveitis and bronchoscopic lung biopsy showed non-caseating epithelioid granuloma. The skin lesions on his lower legs were diagnosed as a specific granuloma of sarcoidosis. He has been treated with topical corticosteroid ointment and the indurated erythema gradually regressed.

\section{Case 2:}

A 65-year-old woman complained cutaneous lesions on the legs. A physical examination showed indurated erythema scattered on the frontal aspects of bilateral legs. Results of laboratory examination revealed slight increase of serum levels of ACE (22.8 IU/L, normal; 8.3 to 21.4) and lysozyme (13 $\mu \mathrm{g} / \mathrm{mL}$; normal 5 to 10.2 ). 
Biopsy specimen revealed non-caseating epithelioid granuloma located in the subcutaneous tissues (Fig. 2).

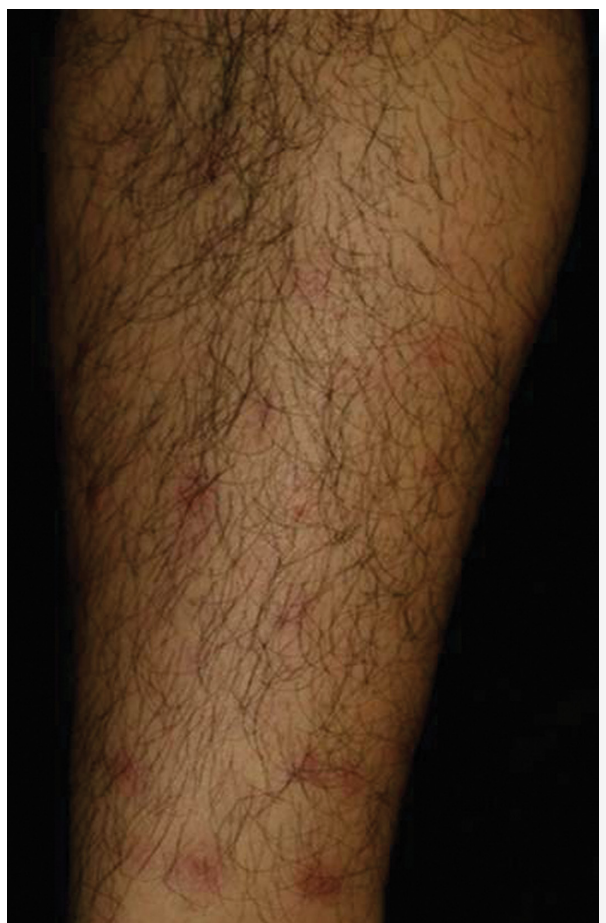

Figure 1a. Numerous erythemaous patches with mild tenderness on the anterior lower leg

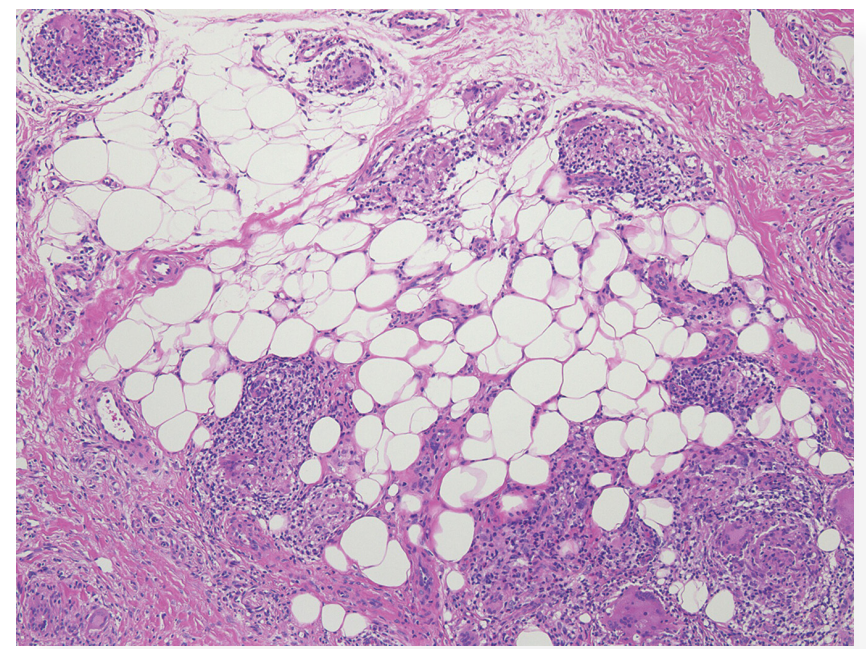

Figure 2. Histological features of Case 2, showing non-caseating epithelioid granuloma in the subcutaneous tissues

\section{Discussion}

Skin manifestations in sarcoidosis are seen in about 20$35 \%$ of patients [2]. Cutaneous lesions have been classified into specific and non-specific manifestations, based on the presence of non-caseating granulomas in the histologic examination. The most common non-specific lesion is EN which histologically shows septal panniculitis without sarcoidal granuloma. Löfgren's syndrome was first recognized as an acute and benign subtype of sarcoidosis, which usually presents the symptoms of EN, acute polyarthritis and bilateral
Chest X-ray revealed bilateral hilar lymphadenopathy. Ocular involvement was not seen.

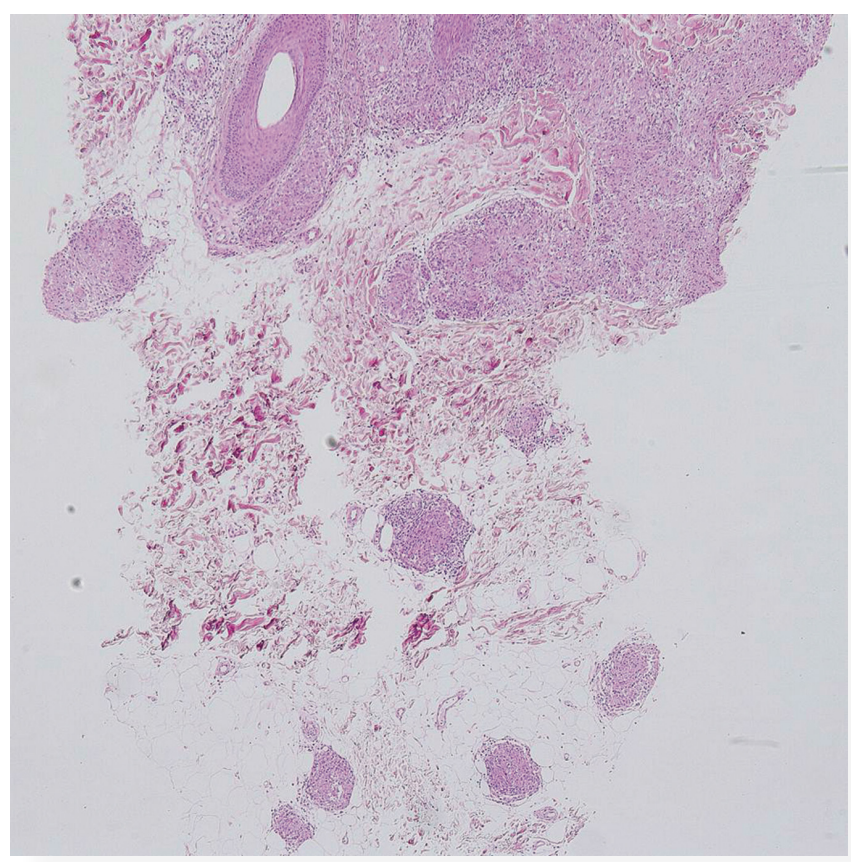

Figure 1b. Histopathological findings reveal circumscribed non-caseating epithelioid granulomas in the mid-dermis to subcutaneous tissues. (H\&E stain, $\times 100)$

hilar lymphadenopathy [3]. It is currently considered as a variant of sarcoidosis. A skin biopsy is necessary to make a distinction between specific sarcoidal granuloma and panniculitis of Löfgren's syndrome [4].

By contrast, EN-like eruption is a specific manifestation of sarcoidosis. Apart from EN in Löfgren's syndrome, sarcoidal granuloma is histologically detected. EN-like eruption associated with sarcoidosis has been reported in Japan; however, we can find only a few reports in English literatures $[5,6]$. EN-like eruption was unusual and the frequency was estimated approximately to be $5 \%$ among the cutaneous sarcoidosis in Japan. During these 30 years, we collected 31 cases reported in Japanese and the presented cases herein. 25 out of 31 are females $(80.6 \%)$ and the mean age is 38 years old. Ophthalmic involvements are particularly seen in $23(74.2 \%)$ of patients with EN-like eruptions, in which skin lesions preceded ocular lesions in 10 patients. Skin biopsies reveal the presence of non-caseating epithelioid granuloma in the mid-dermis to subcutaneous tissues. The clinical symptoms such as tenderness and subcutaneous induration tend to be milder than that of non-specific EN lesion. EN-like eruption tends to regress spontaneously [6].

\section{Conclusion}

Because of the high frequency of extra-cutaneous complications, cases presenting EN-like lesions have to be carefully followed up for ocular and lung involvements of sarcoidosis. 


\section{REFERENCES}

1. Heath CR, David J, Taylor SC: Sarcoidosis: are there differences in your skin of color patients? J Am Acad Dermatol. 2012;66:121. e1-14.

2. Fernandez FE, McDonnell J: Cutaneous sarcoidosis: differential diagnosis. Clin Dermatol. 2007;25:276-87.

3. Mañá J, Gómez-Vaquero C, Montero A, Salazar A, Marcoval J, Valverde J et al: Löfgren's syndrome revisited: a study of 186 patients. Am J Med. 1999;107:240-5.
4. Kumar G, Kumar N: All red is not always bacterial cellulitis: a case of Löfgren's syndrome. WMJ. 2010;109:31-3.

5. Okamoto H, Mizuno K, Imamura S, Nagai S, Izumi T: Erythema nodosum-like eruption in sarcoidosis. Clin Exp Dermatol. 1994;19:507-10.

6. Takenoshita H, Yamamoto T: Erythema nodosum-like cutaneous lesions of sarcoidosis showing livedoid changes in a patient with sarcoidosis and Sjogren syndrome. Eur J Dermatol. 2010;20:640-1.

Copyright by Taeko Nakamura-Wakatsuki, et al. This is an open access article distributed under the terms of the Creative Commons Attribution License, which permits unrestricted use, distribution, and reproduction in any medium, provided the original author and source are credited. 